\title{
Identifying Supermassive Black Hole Binaries with Broad Emission Line Diagnosis
}

\section{Citation}

Shen, Yue, and Abraham Loeb. 2010. "IDENTIFYING SUPERMASSIVE BLACK HOLE BINARIES WITH BROAD EMISSION LINE DIAGNOSIS." The Astrophysical Journal 725 (1): 249-60. https:// doi.org/10.1088/0004-637x/725/1/249.

\section{Permanent link}

http://nrs.harvard.edu/urn-3:HUL.InstRepos:41393152

\section{Terms of Use}

This article was downloaded from Harvard University's DASH repository, and is made available under the terms and conditions applicable to Other Posted Material, as set forth at http:// nrs.harvard.edu/urn-3:HUL.InstRepos:dash.current.terms-of-use\#LAA

\section{Share Your Story}

The Harvard community has made this article openly available.

Please share how this access benefits you. Submit a story.

Accessibility 


\title{
IDENTIFYING SUPERMASSIVE BLACK HOLE BINARIES WITH BROAD EMISSION LINE DIAGNOSIS
}

\author{
Yue Shen ANd Abraham Loeb \\ Harvard-Smithsonian Center for Astrophysics, 60 Garden Street, MS-51, Cambridge, MA 02138, USA \\ Received 2010 April 6; accepted 2010 September 25; published 2010 November 17
}

\begin{abstract}
Double-peaked broad emission lines in active galactic nuclei may indicate the existence of a bound supermassive black hole (SMBH) binary where two distinct broad-line regions (BLRs) contribute together to the line profile. An alternative interpretation is a disk emitter origin for the double-peaked line profile. Using simple BLR models, we calculate the expected broad line profile for an SMBH binary at different separations. Under reasonable assumptions that both BLRs are illuminated by the two active SMBHs and that the ionizing flux at the BLR location is roughly constant, we confirm the emergence of double-peaked features and radial velocity drifts of the two peaks due to the binary orbital motion. However, such a clear double-peaked feature only arises in a particular stage of the binary evolution when the two black holes (BHs) are close enough such that the lineof-sight orbital velocity difference is larger than the FWHM of the individual broad components, while the two BLRs are still mostly distinct. Prior to this stage, the velocity splitting due to the orbit motion of the binary is too small to separate the emission from the two BLRs, leading to asymmetric broad line profiles in general. When the two BHs are even closer such that the two BLRs can no longer be distinct, the line profile becomes more complex and the splitting of the peaks does not correspond to the orbital motion of the binary. In this regime, there are no coherent radial velocity drifts in the peaks with time. Asymmetric line profiles are probably a far more common signature of binary SMBHs than double-peaked profiles. We discuss the temporal variations of the broad line profile for binary SMBHs and highlight the different behaviors of reverberation mapping in the binary and disk emitter cases, which may serve as a feasible tool to disentangle these two scenarios.
\end{abstract}

Key words: black hole physics - galaxies: active - quasars: general - surveys

Online-only material: color figures

\section{INTRODUCTION}

Binary supermassive black holes (SMBHs) are generic outcomes of hierarchical galaxy mergers (e.g., Colpi \& Dotti 2009, and references therein). A couple of galactic-scale binary active galactic nuclei (AGNs) were reported based on spatially resolved imaging and spectroscopy (e.g., Komossa et al. 2003; Bianchi et al. 2008; Comerford et al. 2009b). These binaries are at projected separations of the order of kpc, below which it is difficult to spatially resolve both SMBHs at cosmological distances. While in principle radio interferometry can resolve close binaries down to millarcsecond resolution, it requires both black holes (BHs) to be radio sources and so far there is only one sub-kpc binary candidate detected in the radio (Rodriguez et al. 2006).

Characteristic velocity offsets or double-peaked features in AGN emission line (both broad and narrow lines) profiles have been invoked to indicate the presence of a binary SMBH, even if its spatial extent is not resolved. While this idea is not new (e.g., Heckman et al. 1981; Gaskell 1983; Peterson et al. 1987), it only recently became feasible to search for such binary candidates in a systematic way using large statistical samples with good spectral quality (most notably the Sloan Digital Sky Survey ${ }^{1}$ (SDSS) samples). Increasingly larger spectroscopic samples are starting to offer a unique opportunity to search for candidate binary SMBHs based on their spectral properties (e.g., Zhou et al 2004; Bonning et al. 2007; Komossa et al. 2008; Comerford et al. 2009a; Boroson \& Lauer 2009; Liu et al. 2010b; Smith et al. 2009; Shields et al. 2009; Wang et al. 2009; Xu \& Komossa 2009). This is an important first

\footnotetext{
1 http://www.sdss.org/
}

step toward quantifying the frequency of binary SMBHs at various separations and providing constraints on hierarchical galaxy merger models and predictions for future low-frequency gravitational wave experiments such as the Laser Interferometer Space Antenna ${ }^{2}$ (LISA).

While it has become routine to select candidate binaries from large spectroscopic data sets, these candidates are less secure than spatially resolved cases. The usual difficulty involves the poorly understood emission-line region geometry and kinematics even for single BHs, which may mimic a binary system. It is rather difficult to rule out one or the other based on a singleepoch spectrum alone. In the case of kpc separation, binary SMBHs, high spatial resolution imaging, and spectroscopy may potentially resolve both active BHs and therefore confirm the binary nature (e.g., Liu et al. 2010a). For gravitationally bound binary SMBHs ( $\lesssim 10 \mathrm{pc}$ ), spectral monitoring and reverberation mapping may disentangle the binary scenario from its alternatives (e.g., Gaskell 1983, 2010; Eracleous et al. 1997; Gezari et al. 2007). At even smaller separations, Loeb (2010) suggested that the time dependence of the broad lines due to orbital motion can be detected in binaries on the verge of entering the gravitational wave dominated inspiral.

Given the importance of binary SMBHs for galaxy formation models and future low-frequency gravitational wave detection experiments, it is crucial to understand the dynamics and geometry of emission-line regions in a binary system and to identify such binaries in a systematic way. However, despite ongoing efforts (e.g., Escala et al. 2005; Dotti et al. 2006, 2007; Mayer et al. 2007; Bogdanović et al. 2008; Cuadra et al. 2009), it is still challenging to explore the parameter space in detail in 

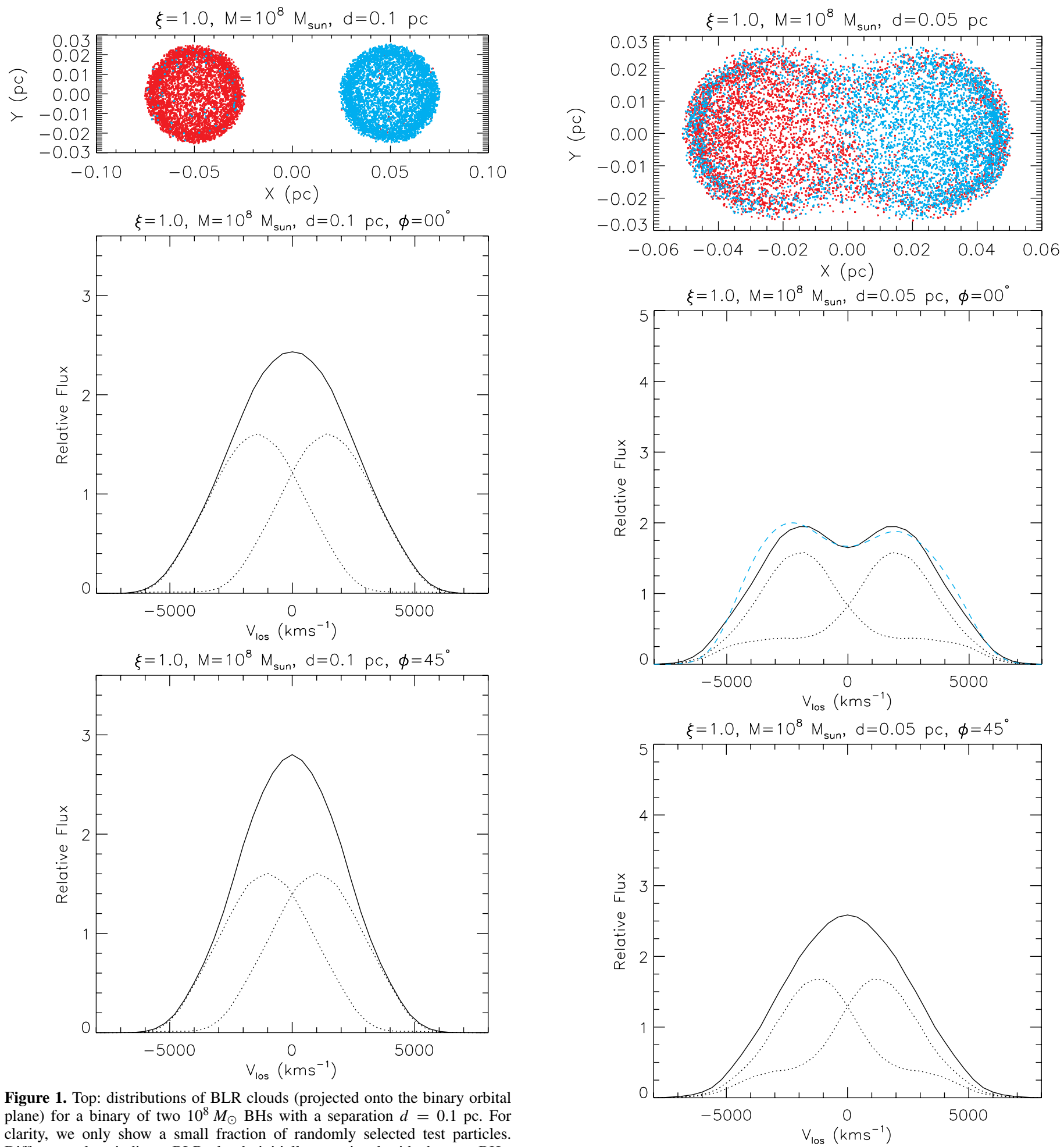

Figure 1. Top: distributions of BLR clouds (projected onto the binary orbital plane) for a binary of two $10^{8} M_{\odot}$ BHs with a separation $d=0.1$ pc. For clarity, we only show a small fraction of randomly selected test particles. Different colors indicate BLR clouds initially associated with the two BHs. The rotation of the binary is counterclockwise. The observer is located in the $x y$ plane at $y=+\infty$, and the radial velocities of the two BHs are maximal at this phase. The few clouds that become mixed with the other BH were initially on highly eccentric orbits with large apocenter or on hyperbolic orbits which travel to the vicinity of the other BH later. We did not remove such clouds in our simulation as they have essentially no effect on the derived line profile. Middle: line profile when the radial velocities of the two BHs are maximal (orbital phase angle $\phi=0^{\circ}$ ). The dotted lines are individual contributions from the two BHs. Bottom: line profile after $1 / 8$ of the orbital period $\left(\phi=45^{\circ}\right)$.

(A color version of this figure is available in the online journal.)

gure 2. Distributions of BLR clouds (projected onto the binary orbital plane) and line profiles for a binary of two $10^{8} M_{\odot}$ BHs with a separation $d=0.05 \mathrm{pc}$. Notations are the same as in Figure 1. The cyan dashed line in the middle panel shows a disk emitter model (see the text for details).

(A color version of this figure is available in the online journal.)

numerical simulations with the adequate dynamical range and the necessary input physics. On the other hand, observational constraints on the dynamics and geometry of AGN emissionline regions have not yet converged to provide reliable inputs for numerical simulations. Here, we use simple toy models to 

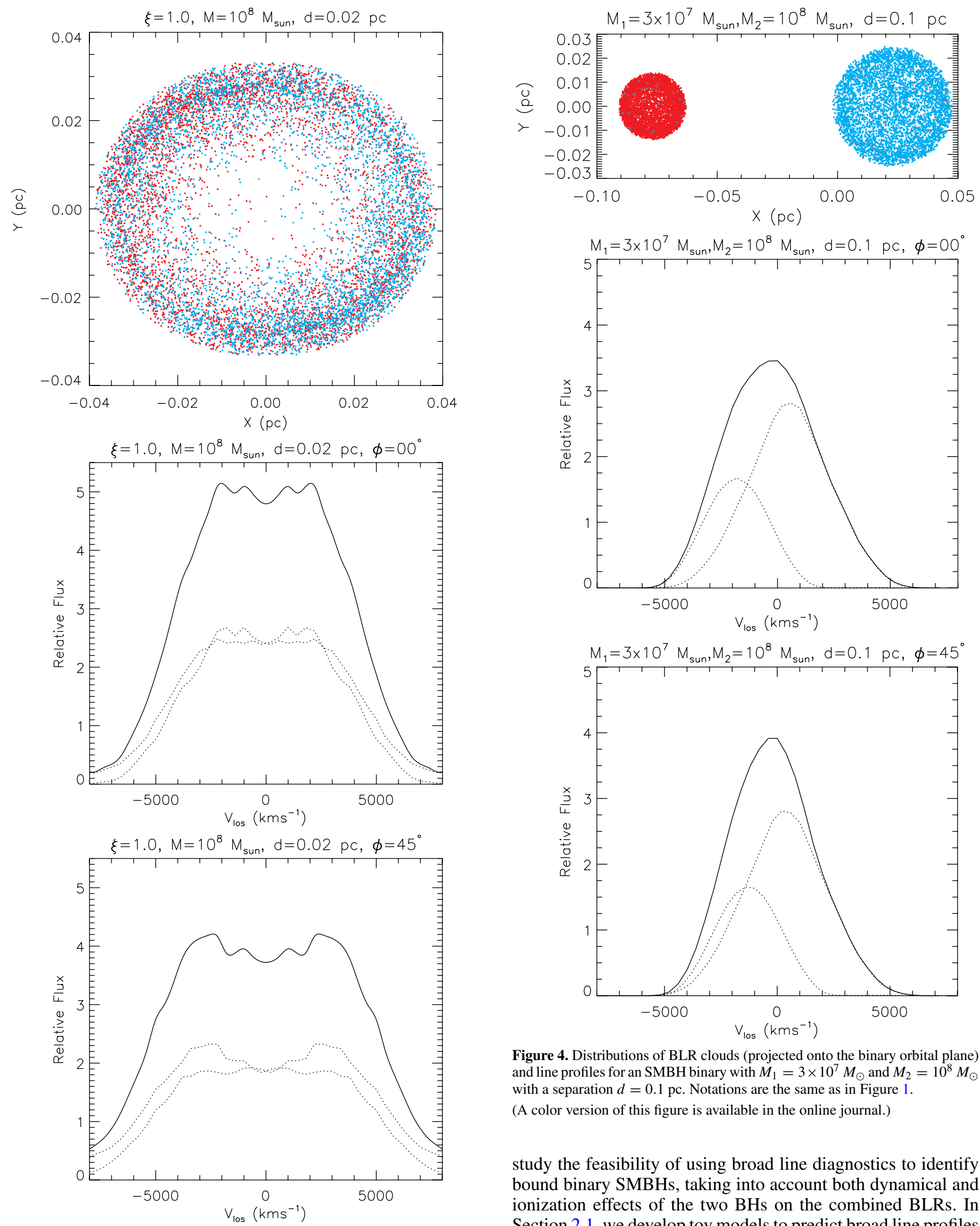

Figure 4. Distributions of BLR clouds (projected onto the binary orbital plane) and line profiles for an SMBH binary with $M_{1}=3 \times 10^{7} M_{\odot}$ and $M_{2}=10^{8} M_{\odot}$ with a separation $d=0.1 \mathrm{pc}$. Notations are the same as in Figure 1.

(A color version of this figure is available in the online journal.)

study the feasibility of using broad line diagnostics to identify bound binary SMBHs, taking into account both dynamical and ionization effects of the two BHs on the combined BLRs. In Section 2.1, we develop toy models to predict broad line profiles under various circumstances. In Section 3, we discuss the

Figure 3. Distributions of BLR clouds (projected onto the binary orbital plane) and line profiles for a binary of two $10^{8} M_{\odot}$ BHs with a separation $d=0.02 \mathrm{pc}$. Notations are the same as in Figure 1.

(A color version of this figure is available in the online journal.) Finally, we discuss our results in Section 4. Unless otherwise 

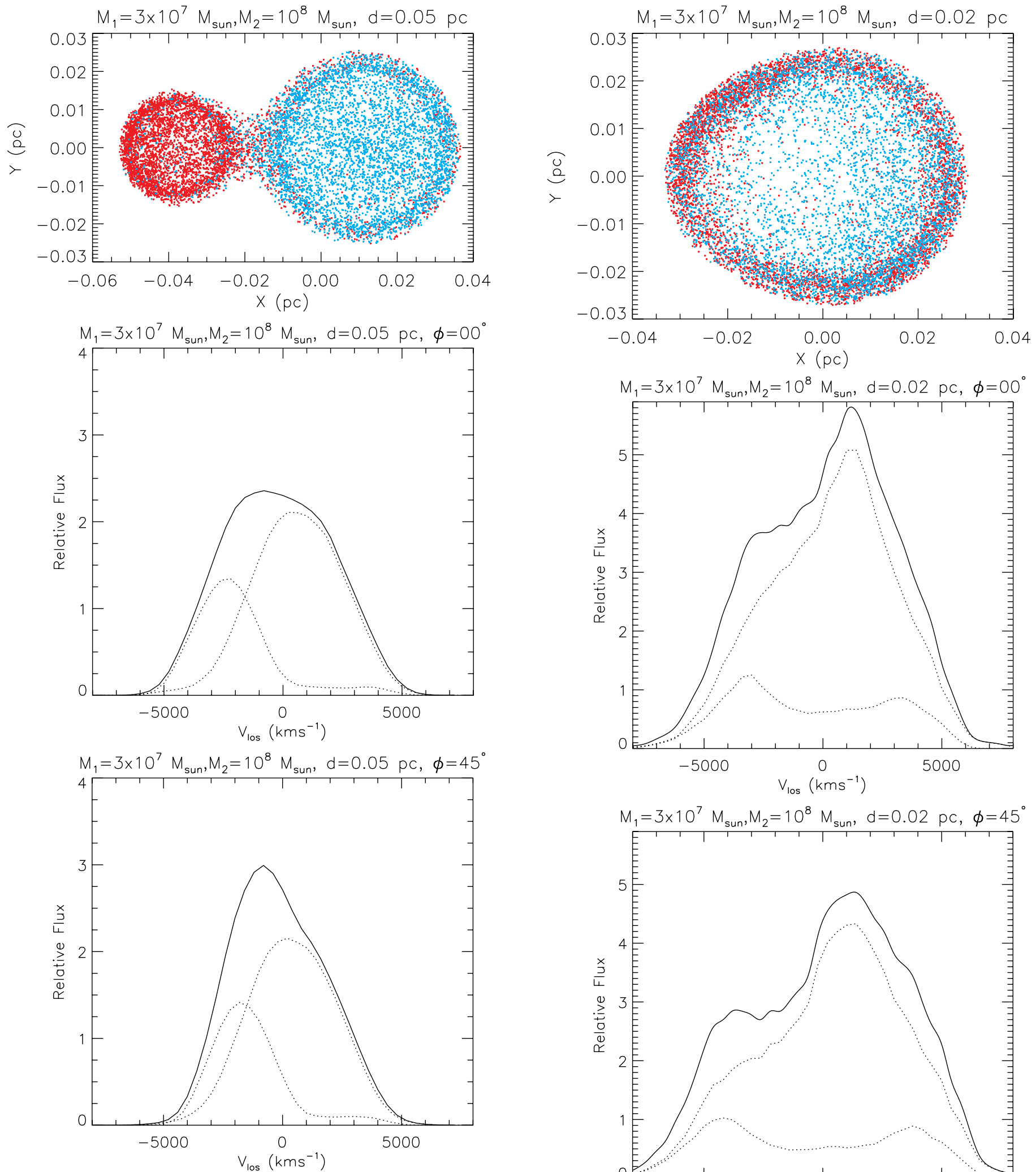

Figure 5. Distributions of BLR clouds (projected onto the binary orbital plane) and line profiles for an SMBH binary with $M_{1}=3 \times 10^{7} M_{\odot}$ and $M_{2}=10^{8} M_{\odot}$ with a separation $d=0.05 \mathrm{pc}$. Notations are the same as in Figure 1.

(A color version of this figure is available in the online journal.)

stated, we will use $\mathrm{H} \beta$ as the fiducial emission line since this is the best-studied broad line in reverberation mapping studies from which characteristic BLR properties are best determined.
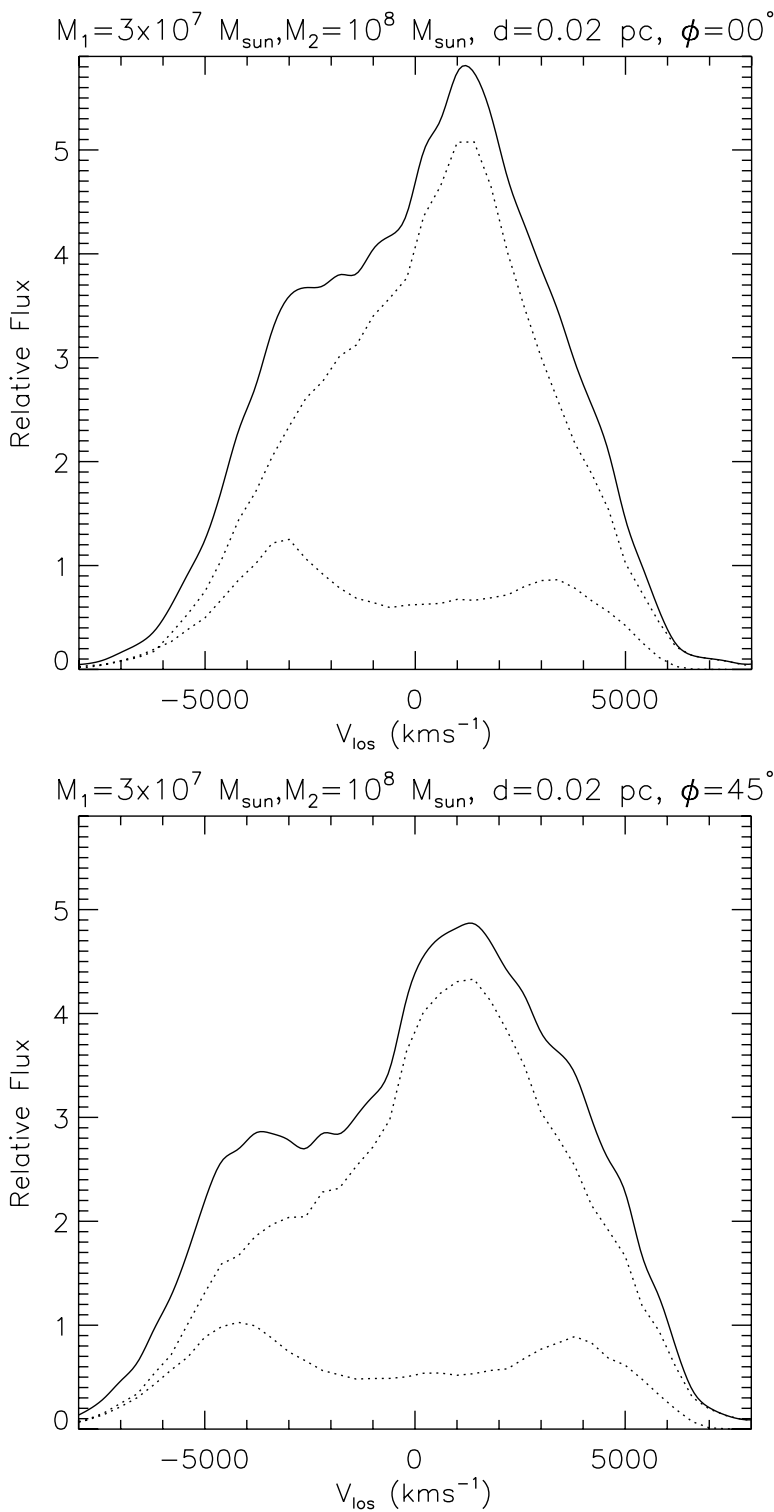

Figure 6. Distributions of BLR clouds (projected onto the binary orbital plane) and line profiles for an SMBH binary with $M_{1}=3 \times 10^{7} M_{\odot}$ and $M_{2}=10^{8} M_{\odot}$ with a separation $d=0.02 \mathrm{pc}$. Notations are the same as in Figure 1.

(A color version of this figure is available in the online journal.) 

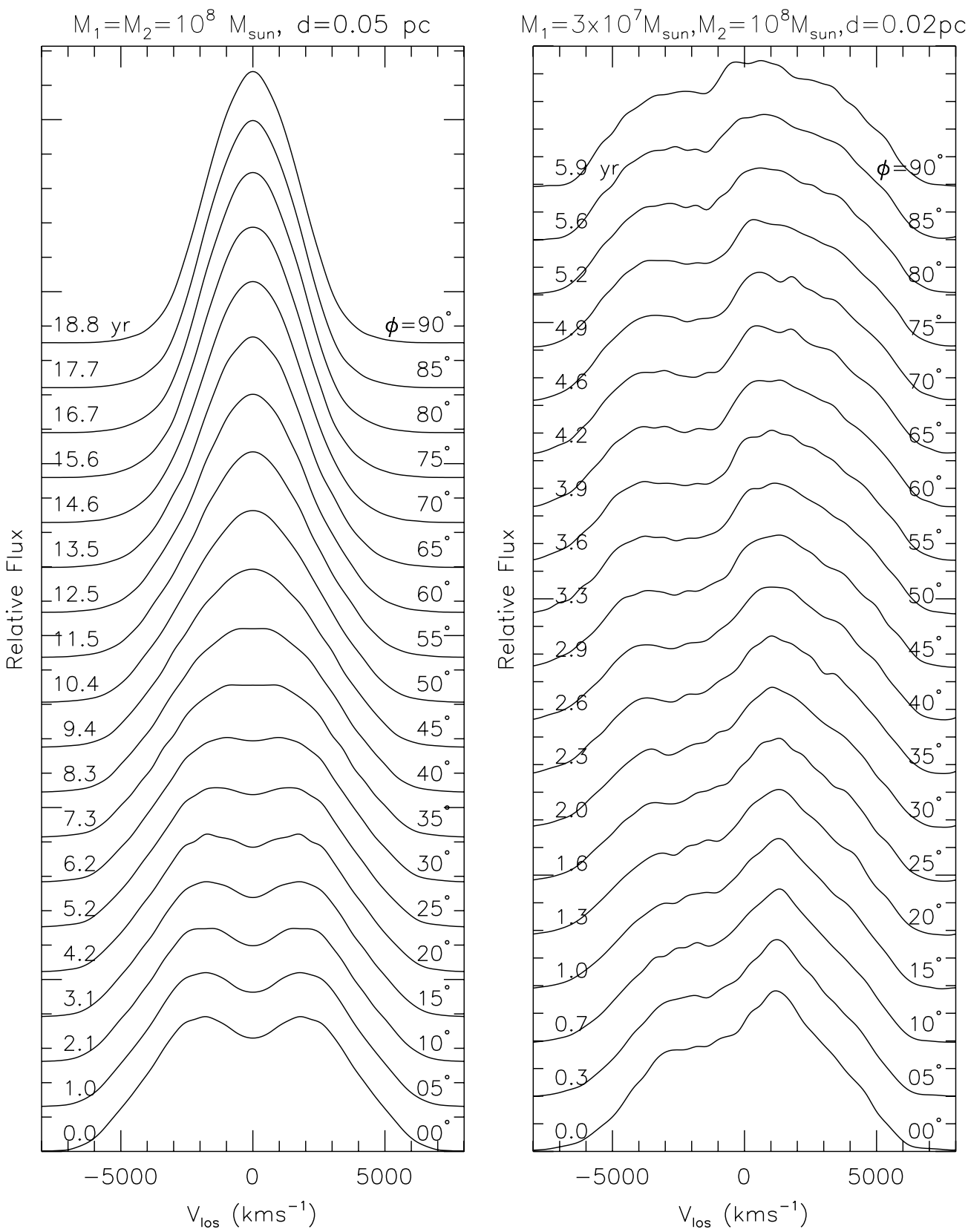

Figure 7. Time series of the radial velocity drifts in the line profile for an edge-on binary SMBH and for $1 / 4$ of the binary orbital period. Left: a binary with $M_{1}=M_{2}=10^{8} M_{\odot}, d=0.05 \mathrm{pc}$, and $P \approx 75 \mathrm{yr}$ (see Figure 2). The two BLRs are distinct in this case and the drifts in radial velocities of the two peaks are apparent. Right: a binary with $M_{1}=3 \times 10^{7} M_{\odot}, M_{2}=10^{8} M_{\odot}, d=0.02 \mathrm{pc}$, and $P \approx 23.5 \mathrm{yr}$ (see Figure 6). The two BLRs are no longer distinct in this case and there are no coherent drifts in the radial velocities of the two peaks.

\section{BROAD EMISSION LINE PROFILES}

We consider the broad line profiles for bound binary SMBHs where the dynamics is dominated by the gravitational potential of the two BHs, assuming both BHs are active and have their own BLRs. We also assume that the two BHs are corotating on a circular orbit. We are interested in binary SMBHs with comparable masses $\left(0.3 \leqslant \xi \equiv M_{1} / M_{2} \leqslant 1\right)$, since binaries with extreme mass ratios are either difficult to detect with broad line diagnosis (if line emission is proportional to $\mathrm{BH}$ mass) or difficult to form due to the extended dynamical friction time of the galaxy merger. For simplicity, we also assume a fixed Eddington ratio $\lambda_{\text {Edd }}=0.1$ (e.g., Shen et al. 2008), but we note that in practice the two active BHs could have different
Eddington ratios. For demonstration purposes we will only show binary examples with an edge-on view (Figures 1-9). The effect of inclination $I$ is to reduce the radial velocities by $\sin I$. We use $\phi$ to denote the binary orbital phase with $\phi=0^{\circ}$ when the radial velocities of the two BHs are maximal.

If both BHs have their own distinct BLR and each BLR is corotating along with its $\mathrm{BH}$ in the binary orbit, we expect to see two time-varying velocity components in the broad line profile. The velocity splitting of the two components depends on the binary orbit separation and the BLR sizes. When the orbital separation is large, the velocity splitting of the two broad line components is small compared with the broad line width and the two components will blend with each other in the spectrum. When the two BHs come closer, the velocity splitting gets wider 

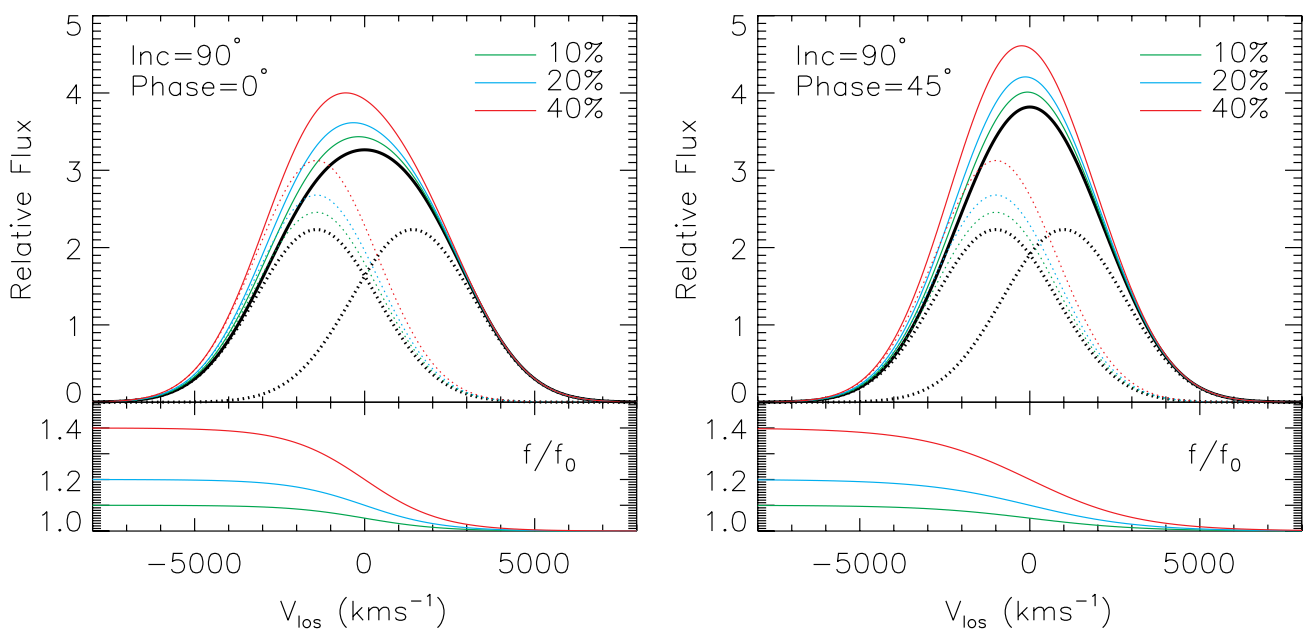

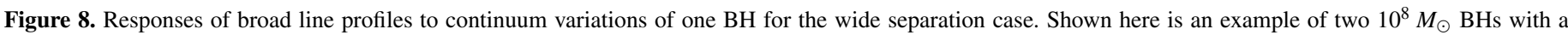

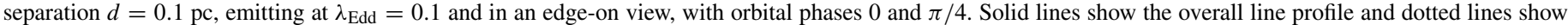

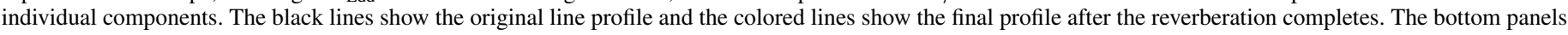

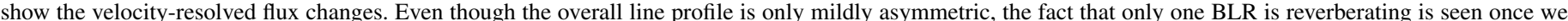
decompose the broad line profile into two Gaussian components.

(A color version of this figure is available in the online journal.)
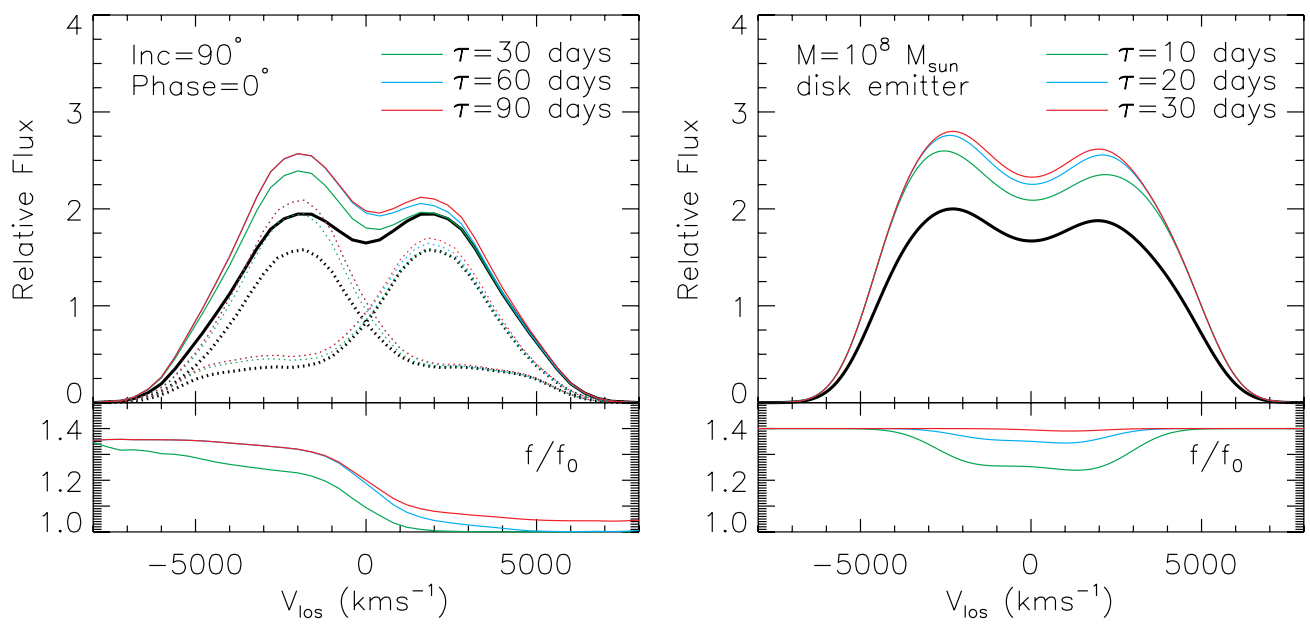

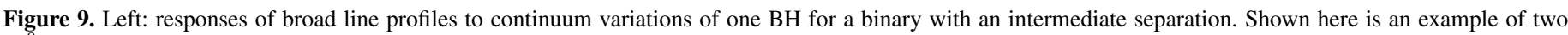

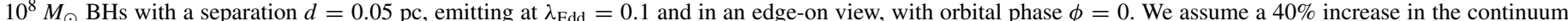

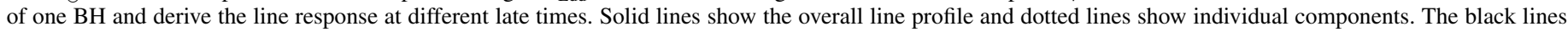

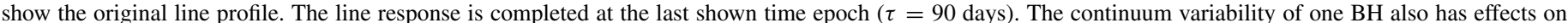

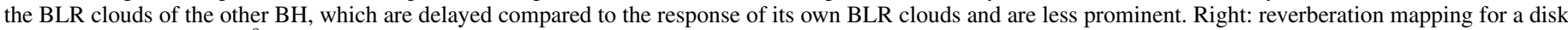

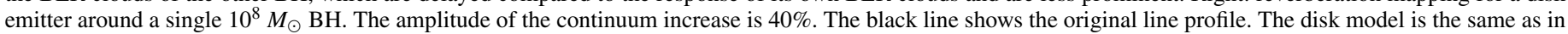
the middle panel of Figure 2. The line response is completed at the last shown time epoch ( $\tau=30$ days).

(A color version of this figure is available in the online journal.)

and a double-peak profile may emerge. When the two BHs come even closer, both BLRs are dynamically influenced by the two BHs and so some BLR material becomes circumbinary, leading to a more complex velocity structure in the combined line profile. The velocity peaks in the broad line, if any, will not simply correspond to the orbital motion of the binary in this case. Eventually, the two BHs will get so close that they dynamically affect the BLR clouds like a single BH, and the broad line profile may become single-peaked again. These simple arguments suggest that a clear double-peaked broad line profile may only arise during a particular stage of the binary orbit evolution, where the orbital velocity of the binary is large enough to split the two peaks and both BLRs are still mostly distinct.
Denoting the separation of the binary as $d$ and the BLR radius in a single $\mathrm{BH}$ as $R_{\mathrm{BLR}}$, the criterion for both BLRs to be bound to their own BHs is $R_{\mathrm{BLR}} \lesssim 0.5 d$. For a single $\mathrm{BH}$, assuming the BLR is photoionized by the continuum luminosity from the central $\mathrm{BH}$, the $R_{\mathrm{BLR}}-L$ relation (assuming $R_{\mathrm{BLR}} \propto L^{1 / 2}$ ) derived from reverberation mapping (e.g., Kaspi et al. 2000; Peterson et al. 2004; McLure \& Jarvis 2002; Kaspi et al. 2007) gives

$$
R_{\mathrm{BLR}} \approx 2.2 \times 10^{-2}\left(\frac{\lambda_{\mathrm{Edd}}}{0.1}\right)^{1 / 2}\left(\frac{M_{\bullet}}{10^{8} M_{\odot}}\right)^{1 / 2} \mathrm{pc}
$$

where $M_{\bullet}$ is the $\mathrm{BH}$ mass and $\lambda_{\mathrm{Edd}}$ is the Eddington ratio of 
the broad line $\mathrm{AGN}{ }^{3}$ This $R_{\mathrm{BLR}}-L$ scaling implies that the ionization parameter, $U \propto L / r^{2}$, is roughly constant for a particular line species (assuming constant electron density). The orbital period for a circular orbit at this location is

$$
t_{\text {orb }}=30\left(\frac{\lambda_{\text {Edd }}}{0.1}\right)^{3 / 4}\left(\frac{M_{\bullet}}{10^{8} M_{\odot}}\right)^{1 / 4} \mathrm{yr} .
$$

Assuming the BLR is virialized, the FWHM of a single broad component is

$$
\begin{aligned}
v_{\mathrm{FWHM}}= & \left(\frac{G M_{\bullet}}{f R_{\mathrm{BLR}}}\right)^{1 / 2} \\
\approx & 4200\left(\frac{\lambda_{\mathrm{Edd}}}{0.1}\right)^{-1 / 4} \\
& \times\left(\frac{M_{\bullet}}{10^{8} M_{\odot}}\right)^{1 / 4}\left(\frac{f}{1.4}\right)^{-1 / 2} \mathrm{~km} \mathrm{~s}^{-1},
\end{aligned}
$$

where $f$ is the virial coefficient accounting for our ignorance of the BLR geometry and is of order unity. A recent determination of $f$ based on the reverberation mapping $\mathrm{BH}$ masses and $\mathrm{BH}$ masses from the $M_{\bullet}-\sigma$ relation gives $f \approx 1.4$ (Onken et al. 2004). ${ }^{4}$

If the two BLRs are distinct and simply corotate along with their own BHs in a binary system, the line-of-sight (LOS) velocity splitting of the two broad line peaks changes periodically in time $t$ :

$$
\begin{aligned}
v_{\mathrm{los}}= & {\left[\frac{G M_{\mathrm{tot}}}{d}\right]^{1 / 2} \sin I \sin (2 \pi t / P) } \\
= & 6300\left(\frac{M_{\mathrm{tot}}}{10^{8} M_{\odot}}\right)^{1 / 2}\left(\frac{d}{0.01 \mathrm{pc}}\right)^{-1 / 2} \\
& \times \sin I \sin (2 \pi t / P) \mathrm{km} \mathrm{s}^{-1},
\end{aligned}
$$

where $M_{\mathrm{tot}}=M_{1}+M_{2}$ is the total mass of the two BHs, $I$ is the inclination of the binary orbital plane relative to the LOS, and

$$
\begin{aligned}
P & \equiv 2 \pi d^{3 / 2}\left(G M_{\mathrm{tot}}\right)^{-1 / 2} \\
& =9.5 \times 10^{3}\left(\frac{d}{1 \mathrm{pc}}\right)^{3 / 2}\left(\frac{M_{\mathrm{tot}}}{10^{8} M_{\odot}}\right)^{-1 / 2} \mathrm{yr}
\end{aligned}
$$

is the orbital period of the binary.

From these simple calculations (1)-(4) we can investigate how well separated the double components could be without violating the assumption that each BLR is mostly under the influence of only one BH. In order to see a clear doublepeaked feature we require $v_{\text {los, } \max } \gtrsim v_{\text {FWHM }}$, while in order to have distinct BLRs we need $R_{\mathrm{BLR}} \lesssim d / 2$. Assuming $\xi \equiv M_{1} / M_{2} \leqslant 1$ these two criteria imply

$0.044\left(\frac{M_{2}}{10^{8} M_{\odot}}\right)^{1 / 2} \mathrm{pc} \lesssim d \lesssim 0.063\left(\frac{1+\xi}{2}\right)\left(\frac{M_{2}}{10^{8} M_{\odot}}\right)^{1 / 2} \mathrm{pc}$.

Therefore, there is only a narrow window of separation (as well as mass ratio $\xi$ ) within which a double-peaked broad line profile may emerge without violating the assumption that both BLRs are distinct.

\footnotetext{
3 The $R_{\mathrm{BLR}}-L$ relation is usually calibrated using the rest-frame $5100 \AA$ continuum luminosity. We have adopted a bolometric correction

$\mathrm{BC}_{5100 \AA}=10$ to convert the continuum luminosity to bolometric luminosity.

4 Note that we have used a different definition of the virial coefficient from others. The coefficient $f$ here corresponds to the coefficient $\epsilon$ in Onken et al. (2004).
}

\subsection{Model Setup}

Next, we investigate the broad line profile for a binary SMBH in a more quantitative manner. First, we describe our approach to model the BLRs of a binary SMBH.

We start with a simple prescription for the BLR around a single $\mathrm{BH}$, where the BLR is assumed to be an assembly of discrete clouds (e.g., Peterson 1997). Then two BHs with their individual BLRs are placed on a circular orbit. We integrate numerically the orbits of individual clouds (treated as test particles) in the circular restricted three-body problem. By imposing an ionization condition (see below), we identify the clouds that will radiate the line emission and determine the line profile based on the LOS velocity distribution of these lineemitting clouds. This procedure is detailed below.

First, we need to specify a model for the BLR around a single $\mathrm{BH}$. Despite decades of research, the detailed structure of BLR is still poorly constrained. The most powerful observational tool to study BLR structure is reverberation mapping. But even with the best-studied reverberation mapping sample, there is still no complete consensus on the general BLR structure (e.g., Denney et al. 2009). Nevertheless, reverberation mapping does provide a characteristic scale for the BLR radius, given by the $R_{\mathrm{BLR}}-L$ relation in Equation (1). In a handful of cases, reverberation mapping of different line species in the same system shows that lines with larger ionization parameters have smaller radii from the central BH (e.g., Peterson \& Wandel 2000) and that the velocity inferred from the line width is consistent with virialized motion.

Motivated by these observations, we adopt the following simple model for the BLR around a single BH. For a single BH, we uniformly populate clouds as test particles within a spherical shell with inner and outer radii $\sqrt{0.8}$ and $\sqrt{1.2}$ of the radius in Equation (1), i.e., the flux or ionization parameter (assuming constant electron density) required to photoionize a particular line species in the BLR clouds is roughly constant within $\pm 20 \%$ (the exact value of the percentage does not change our conclusions). The velocities of those clouds are generated from a Maxwellian distribution whose one-dimensional dispersion is determined from the virial relation, $\sigma=\sqrt{G M_{\bullet} /(3 r)}$, with random orientations. Since it is unphysical to restrict these clouds within a perfectly thin shell, we evolve the Keplerian orbits of these clouds in the single BH system using standard analytical formulae (e.g., Murray \& Dermott 1999). The system quickly establishes a quasi-steady state configuration where the spatial and velocity distributions converge after $\sim t_{\text {orb }}$ (Equation (2)). The resulting quasi-steady-state distributions are taken as the initial cloud distributions around a single $\mathrm{BH} .{ }^{5}$ These clouds have a radial distribution peaked around the initial shell location but also extend to larger and smaller radii, and the Gaussianity of their velocity distribution is mostly preserved. We generate 5000 test particles for each BH. As discussed later, the sporadic snapshots of these test particle trajectories will be combined to compute the cloud distributions in a binary system. The resulting line profiles are very smooth even though the total number of test particles used is modest.

This simple model for the BLR around a single $\mathrm{BH}$ is highly idealized, given the limited understandings of actual

\footnotetext{
5 We do not remove the small fraction $(\sim 10 \%)$ of clouds on hyperbolic orbits in the initial distributions, since such clouds will either be bound in the binary system or will escape to large distances and not contribute to line emission anyway. We verified that removing these clouds does not have any effect on the derived line profiles.
} 
BLR properties. It nevertheless reproduces line profiles and characteristic BLR sizes that are consistent with observations. We have assumed that only clouds at a narrow range of distances from the $\mathrm{BH}$ can be line emitting and constitute the BLR. In reality, the BLR in some AGNs could have a larger spatial extent, as inferred in some (albeit limited) reverberation mapping studies (e.g., Denney et al. 2009; Horne et al 2004, and references therein). This can be understood if the electron density in the BLR varies with radius, so that the flux $\left(\propto r^{-2}\right)$ required to produce the proper ionization parameter also has a larger range in photoionization models. However, given the small scatter in the mean $R_{\mathrm{BLR}}-L$ relation (e.g., Peterson 2010), our fiducial choice of a relatively thin shell geometry for the BLR should be a reasonable approximation at least for the majority of the line-emitting clouds in the BLR. One caveat is that we are not including a significant fraction of clouds that are either far more distant or closer in than the characteristic BLR distance. Such clouds will not be line-emitting in the single BH system because the ionization parameter is either too high or too low. Clouds much closer in will be tightly bound to their $\mathrm{BH}$ and will not contribute to the line emission in the binary system either. However, if there are a large amount of cold clouds orbiting outside the BLR in single BHs, these clouds are easier to become circumbinary and may be ionized by the combined continua from both BHs in the binary system, thus making the double-peaked feature less prominent. Below we proceed with our fiducial model for the BLR around single BHs, and the effects of such a distant cloud reservoir will be further discussed in Section 4.1.

We combine the two $\mathrm{BH}$ plus cloud systems to form a binary on a circular orbit. In the frame corotating with the binary, we derive the instantaneous locations and velocities of these clouds by orbital integrations of the restricted three-body problem. We are only interested in the temporal behaviors over a period that can be monitored on human life timescales, so we integrate the system for a few hundred years sampled with 1000 evenly distributed temporal snapshots. Long-term stability of the cloud orbits is not considered in this paper. ${ }^{6}$ We use a Bulirsch-Stoer integrator (Press et al. 1992) and the relative accuracy tracked by the Jacobi constant was well below $10^{-10}$ over the course of the integration. We then combine all the snapshots as a quasi steady-state configuration of clouds in the binary system, so as to improve the statistics of the cloud distributions. We found that the cloud distributions (in real and velocity space) in individual snapshots are similar (but with much poorer statistics) to those from the combined snapshots, after the initial $\sim 10 \%$ of integration time, i.e., the velocity distribution of BLR clouds quickly establishes a quasi-equilibrium in much less than one binary orbit time. Although clouds are still constantly expelled by the binary afterward, the loss of clouds does not have significant effects on the velocity structure of the BLR over the course of the integration.

Once we have the locations and velocities of the clouds, we determine line-emitting BLR clouds under the assumption that clouds are illuminated by both BHs and that the combined flux (or ionization parameter) required to ionize a BLR cloud is roughly constant (within $\pm 20 \%$, assuming a constant electron

\footnotetext{
6 It is possible that some of these orbits are unstable over longer timescales and the long-lived orbits may have somewhat different spatial and velocity distributions. On the other hand, the individual BLRs are likely non-static and some mechanisms may exist to replenish the clouds continuously (such as a wind from the accretion disk). It is beyond the scope of this paper to take into account these complications, but we note that our integration time may be too short for some of the cases studied here.
}

density). Quantitatively, the locations of these line-emitting clouds satisfy the following constraint:

$$
\begin{aligned}
\frac{0.8}{\left(2.2 \times 10^{-2}\right)^{2}} & \leqslant \frac{\left(\frac{\lambda_{\text {Edd }, 1}}{0.1}\right)\left(\frac{M_{1}}{10^{8} M_{\odot}}\right)}{\left(r_{1} / \mathrm{pc}\right)^{2}} \\
& +\frac{\left(\frac{\lambda_{\text {Edd }, 2}}{0.1}\right)\left(\frac{M_{2}}{10^{8} M_{\odot}}\right)}{\left(r_{2} / \mathrm{pc}\right)^{2}} \leqslant \frac{1.2}{\left(2.2 \times 10^{-2}\right)^{2}}
\end{aligned}
$$

where $r_{1}$ and $r_{2}$ are the distances to the two BHs. This condition follows the photoionization criterion for single $\mathrm{BHs}$ in Equation (1).

Based on the procedure outlined above, we derive the broad line profile for binary SMBHs at any orbital phase and hence the temporal variation of line profile due to the orbital motion of the binary.

Figures 1-3 show several examples for an equal-mass binary with $M_{1}=M_{2}=10^{8} M_{\odot}$, and separations of $d=$ $0.1,0.05,0.02 \mathrm{pc}$, with integration time 2,6 and 22 times the binary orbital period given by Equation (5), respectively. Only the intermediate separation case $(d=0.05 \mathrm{pc})$ satisfies the constraint of Equation (6). As the two BHs come closer, their BLRs become less distinct and eventually the two BHs start to affect both BLRs in terms of their dynamics and illumination. In the example with the closest separation (e.g., Figure 3), there is practically a single BLR surrounding the two BHs and the velocity structure of these BLR clouds are more complex than in the distinct BLR cases. Many BLR clouds are now on circumbinary orbits (such as horseshoe or tadpole-type orbits around the triangular Lagrangian points) and there are no coherent radial velocity shifts in the two peaks due to the orbital motion of the binary.

Figures 4-6 show several examples for a non-equal-mass binary with $M_{1}=3 \times 10^{7} M_{\odot}$ and $M_{2}=10^{8} M_{\odot}$ and separations of $d=0.1,0.05,0.02 \mathrm{pc}$, where the BLR clouds associated with each individual $\mathrm{BH}$ are given a flux weight proportional to the $\mathrm{BH}$ mass. The integration times are 2, 6 and 22 times the binary orbital period, respectively. Again, only the intermediate separation case $(d=0.05 \mathrm{pc})$ satisfies the constraint of Equation (6). The qualitative difference from the equal binary case is the increase in line asymmetry and decrease in the prominence of the double peaks (see Popovic et al. 2000, for some similar line profiles generated with different model prescriptions for the binary BLRs).

\subsection{Disk Emitters}

An alternative interpretation of double-peaked broad line profiles is the disk emitter scenario, where the anomalous broad line emission originates from a relativistic accretion disk around a single BH (Chen et al. 1989; Eracleous \& Halpern 1994; Eracleous et al. 1995). In this case, the blueshifted and redshifted components originate from the part of the disk moving toward and away from the observer. The disk emitter model has been successful in reproducing the line profile in many double-peaked broad line AGNs (e.g., Chen et al. 1989; Eracleous \& Halpern 1994; Eracleous et al. 1995, 1997; Strateva et al. 2003; Luo et al. 2009). As an example, in the middle panel of Figure 2 we show a disk emitter model for an inclined (inclination $I=25^{\circ}$ ) elliptical disk (with eccentricity $e=0.1$ ) with inner and outer radii of $500 r_{g}$ and $5000 r_{g}$ (where $r_{g}=G M / c^{2}$ 
is the gravitational radius), specific intensity $I_{v} \propto r^{-3}$, an internal turbulent broadening $\sigma=800 \mathrm{~km} \mathrm{~s}^{-1}$, and a major axis orientation of the elliptical disk $\phi_{0}=60^{\circ}$ (Eracleous et al. 1995 ) around a $\mathrm{BH}$ with $M=10^{8} M_{\odot}$. The disk emitter model clearly shows a double-peaked profile, resembling that for an SMBH binary. But the temporal variations of the broad line in the disk emitter scenario are different from those for the binary SMBH scenario, as we discuss next.

\section{TEMPORAL VARIATIONS}

\subsection{Radial Velocity Drifts}

A definitive signature of a binary SMBH is the time drift in the radial velocities of the decomposed two components, as resulting from the orbital motion of the two BHs. Unfortunately, the typical orbital time is usually much longer than a few years, and in order to detect radial velocity drifts of the double peaks the two BLRs must be distinct. From the previous sections, we know that the optimal configuration to detect such a binary is when the two BLRs are just touching each other, such that the two BLRs are still mostly distinct while at the same time the velocity splitting of the two components is larger than the line width. In the case of two equal mass BHs, substituting $d=2 R_{\mathrm{BLR}}$ into Equation (5), where $R_{\mathrm{BLR}}$ is given by Equation (1), we have $P_{\text {opt }} \sim 62\left(M_{\bullet} / 10^{8} M_{\odot}\right)^{1 / 4} \mathrm{yr}$. This means it is less challenging to detect radial velocity changes in low-mass SMBH binaries by spectral monitoring. In the case of two $M_{\bullet}=10^{6} M_{\odot}$, the time span between double-peaked and single-peaked profiles is only $P_{\text {opt }} / 4 \sim 5$ yr. The limitation of low-mass binaries is that they cannot be easily observed out to high redshifts due to their relatively low luminosities. Nevertheless, these low-mass systems provide good test cases in the nearby universe.

Figure 7 shows changes in the overall line profile due to the orbital motion of the binary for an intermediate separation case (the example in Figure 2) and for a close separation case (the example in Figure 6). While the radial velocity drifts of the two peaks in the intermediate separation case are apparent, there are no coherent drifts in the radial velocities of the two peaks in the close separation case when the two BLRs are no longer distinct (see the upper panel of Figure 6). The non-detection of coherent radial velocity drifts in some of the double-peaked broad line AGNs may then rule out the existence of two distinct BLRs (e.g., Eracleous et al. 1997; Gezari et al. 2007), but cannot rule out the possibility of a close SMBH binary surrounded by a circumbinary BLR, as pointed out in Eracleous et al. (1997). Therefore, additional tests are required to distinguish the binary and disk emitter scenarios in these $\operatorname{cases}^{7}$ (see below).

\subsection{Reverberation Mapping}

A better way to distinguish the binary scenario and the disk emitter scenario is reverberation mapping ${ }^{8}$ (e.g., Peterson et al. 1987; Gaskell 2010). Due to the different geometries of the BLR in the two scenarios, the response of the line to the variations in the continuum follows different patterns.

\footnotetext{
7 In addition to the reverberation mapping method discussed in Section 3.2, there could be variations due to the orbital motion (Doppler effect) of the continuum emitting region of the accretion disk around each $\mathrm{BH}$ (B. Kocsis \& A. Loeb 2010, in preparation).

8 In principle, one could argue that independent variations in the two velocity components of the broad line may be sufficient to distinguish the binary interpretation from disk emitters (e.g., Gaskell 2010). However, reverberation mapping gives a cleaner signature since the variation in the broad line is known to be caused by the variation in the continuum and not by changes in the structure of the BLR.
}

In the case of wide separation binaries, each BLR is illuminated by its own $\mathrm{BH}$ and therefore only responds to the luminosity variations of its $\mathrm{BH}$. We demonstrate this case with the example of two $10^{8} M_{\odot}$ BHs with a separation $0.1 \mathrm{pc}$. The orbital time of the binary is $\sim 200 \mathrm{yr}$, while the typical variability timescale for these BH masses is a few months (e.g., Peterson 1997). Once the continuum of one of the BHs varies, it takes $\sim 1$ month for the associated BLR emission to vary, and the orbital motion of the binary during this light travel time is negligible. The effects of continuum variations of one $\mathrm{BH}$ on the overall broad line profile are shown in Figure 8 for several variability levels and two orbital phases, after the broad line has completed its reverberation ( $t \gtrsim 35$ days). Since the binary separation is large, the broad line is not double-peaked, but the line response introduces an asymmetry that correlates with the amplitude of the continuum variation. This asymmetry is more prominent at smaller orbital phase angles where the two velocity components overlap less in the spectrum.

In the case of intermediate separation binaries, the two BHs are close enough such that part of the BLRs are illuminated by both BHs. Consider the example of two $10^{8} M_{\odot}$ BHs with a separation $0.05 \mathrm{pc}$, i.e., about twice the size of a single BLR. In this case, we use the configuration of BLR clouds derived in our orbital integrations to compute the line profile changes due to continuum changes of one $\mathrm{BH}$. The orbital time of the binary is $\sim 75 \mathrm{yr}$, hence the orbital motion is negligible compared to the light travel time to the BLR, which is a few months. However, due to the proximity of the two $\mathrm{BHs}$, the continuum variation in one of the BHs also affects the other BLR, although the effect is delayed and less prominent than those on its own BLR. The left panel in Figure 9 demonstrates these effects for an edge-on view and orbital phase $\phi=0^{\circ}$. For comparison, the right panel shows the expected line responses to continuum variations for the same disk emitter model shown in Figure 2 at various times, where the line response is complete at the last epoch. Although the high-velocity wing responds more quickly than the central part of the line (because the former emission originates closer to the $\mathrm{BH}$ ), the two peaks change simultaneously. Even though initially the broad line profiles are similar in the binary case and in the disk emitter case, their subsequent line responses are different: (1) the disk emitter completes the response of the line much faster than the binary due to the proximity of the disk to the central $\mathrm{BH}$; (2) both peaks are varying in proportion to each other in the disk emitter case, while in the binary case one peak has larger amplitude changes than the other; and (3) the two peaks reverberate simultaneously in the disk emitter case, whereas in the binary case one peak reverberates faster than the other.

In the case of close separation binaries where the two BLRs are already merged, the behavior of line response to continuum variations of one of the $\mathrm{BHs}$ is similar to that of the intermediate separation binary, but the relative increase in the two peaks is less discrepant than in the intermediate separation binary. Nevertheless, reverberation mapping will be a useful test here because the tests on radial velocity drifts are inconclusive, as discussed in Section 3.1.

\section{DISCUSSION}

\subsection{Complications in the Realistic Situation}

Our simple prescription for the BLR of an SMBH binary is by no means realistic, especially for the closest separation cases studied here, where there are no longer two distinct 
BLRs. The BLR models we adopted are close to models in which the orbits are random and the dynamics is dominated by the gravitational potential from both BHs. The novelty of our approach is to include the effects of the two BHs in terms of both the clouds dynamics and illumination. Our treatment is more quantitative than earlier qualitative arguments that the doublepeaked components are from individual BLRs (e.g., Gaskell 2010, and references therein).

In our BLR model for single BHs, we assumed a rather simplistic thin shell distribution of clouds. To check the sensitivity of our results to this assumption, we examine the effects of a more extended cloud (not all line-emitting clouds) distribution for single BHs. The upper panel of Figure 10 shows an example for the distribution of clouds around a $10^{8} M_{\odot} \mathrm{BH}$, where the clouds were initially populated between 0.5 and 2 times the characteristic BLR size with a power-law number density $n(r) \propto r^{-1}$ and then relaxed for 30 years using their Keplerian orbits. The initial random velocities of each cloud are assigned using the same scheme described in Section 2.1. The starting configuration for the numerical orbit integration is a more extended distribution of clouds compared to our fiducial model. We integrate an equal-mass binary system $\left(M_{\text {tot }}=2 \times 10^{8} M_{\odot}\right)$ using the new single BLR model and compute emission line profiles in the same way as in Section 2.1. The bottom panel of Figure 10 shows the resulting line profile at phase angle $\phi=0^{\circ}$ and with a binary separation $d=0.05 \mathrm{pc}$. Compared with our previous result, i.e., the middle panel of Figure 2, the doublepeaked feature is much less prominent. This is expected because now the two cloud regions overlap more than in the previous case and more clouds become circumbinary, diluting the distinction between the two BLRs. In practice, the two emission line components will not have equal strength, so some asymmetry is expected in the overall line profile, similar to those shown in Figure 5. Secular changes in the line profile due to the orbital motion of the binary, or velocity resolved reverberations of the blue and red wings of the line, can still be monitored even though the two peaks are blended with each other.

Our toy models confirm the feasibility of using spectral monitoring (for radial velocity drifts) and reverberation mapping (for line responses) to disentangle SMBH binaries from disk emitters. These observations can also be achieved with less expensive narrowband filters. However, there are some practical difficulties with these techniques. The spectral monitoring for radial velocity changes works best for binaries with $d \approx$ $R_{\mathrm{BLR}, 1}+R_{\mathrm{BLR}, 2}$. At larger binary separations, the two broad components will blend with each other in the spectrum, making it less likely to be flagged as a binary candidate, and the orbital period is too long to be detectable. At smaller separations, the BLRs are no longer distinct and the velocity structure becomes more complex with no coherent radial velocity drifts in the peak locations. Thus, the spectral monitoring is suitable for identifying low-mass SMBH binaries $\left(M \sim 10^{6} M_{\odot}\right)$. On the other hand, reverberation mapping is a powerful tool for distinguishing an SMBH binary from a disk emitter, but more complex BLR geometries and kinematics (such as those involving inflows or outflows) will certainly complicate the situation (e.g., Sergeev et al. 1999; Denney et al. 2009).

\subsection{Case Studies: $3 C$ 390.3, SDSS J1536+0441, and Others}

Although there are a few dedicated spectral monitoring programs for double-peaked broad line objects (e.g., Gezari et al. 2007 , and references therein), there is currently no reverberation mapping program for a large sample of such objects. Among the
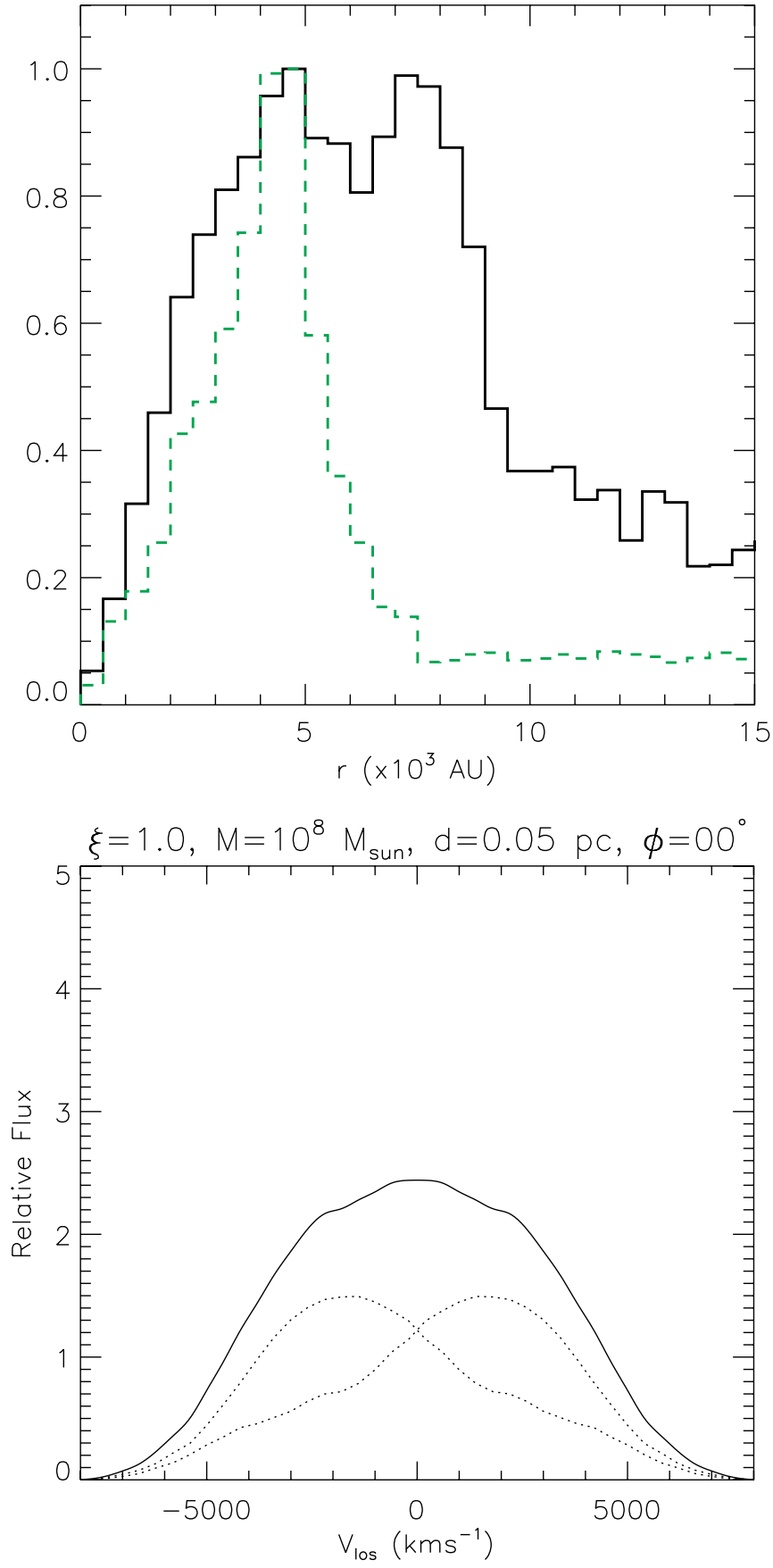

Figure 10. Results for an extended cloud distribution around a single $M=$ $10^{8} M_{\odot}$ BH. Upper: radial distributions of clouds around the single $\mathrm{BH}$ as initial conditions for the numerical orbit integrations. The green dashed line is the fiducial cloud distribution described in Section 2.1, which peaks around the characteristic radius given by Equation (1). The black line is a more extended cloud distribution (see Section 4.1 for details). Bottom: the resulting line profile for the extended cloud distribution and for an equal-mass binary $\left(M_{\mathrm{tot}}=2 \times 10^{8} M_{\odot}\right)$ with a binary separation $d=0.05 \mathrm{pc}$ at phase angle $\phi=0^{\circ}$. The two line components are much less distinct than in the previous case (e.g., Figure 2) because a larger fraction of the outmost BLR clouds are on circumbinary orbits. This tends to dilute the distinction between the two components from BLR clouds orbiting around individual BHs.

(A color version of this figure is available in the online journal.)

$\sim 40$ AGNs that are included in the reverberation mapping sample (Peterson et al. 2004), there are several objects that clearly show double-peaked broad line features. In particular 3C 390.3 is a strong double-peak object with good reverberation mapping 
data (Dietrich et al. 1998). The time-ordered data of this source show that the blueshifted and redshifted components respond to the continuum variations almost simultaneously. Thus, it is more likely that the double-peaked emission originates from a disk rather than from two corotating BLRs in a binary system.

The quasar SDSS J1536+0441 was recently suspected to be a sub-pc binary SMBH (Boroson \& Lauer 2009) because of its double-peaked broad $\mathrm{H} \beta$ line in the SDSS spectrum. It has therefore received much attention (e.g., Chornock et al. 2010; Gaskell 2010; Wrobel \& Laor 2009; Decarli et al. 2009; Tang \& Grindlay 2009). The discovery of an additional redshifted component, most notably in $\mathrm{H} \alpha$ (e.g., Chornock et al. 2010; Lauer \& Boroson 2009), favored a disk emitter origin for the double peaks rather than a binary SMBH. If we nevertheless assume this is a binary SMBH and use constant Eddington ratios $\lambda_{\text {Edd }}=0.1$ for both BHs and the FWHM values measured in Boroson \& Lauer (2009), we get from Equation (3) $M_{1}=8 \times 10^{8} M_{\odot}$ and $M_{2}=2 \times 10^{7} M_{\odot}$ for the red and blue systems, respectively, similar to those reported in Boroson \& Lauer (2009). We also derive BLR sizes $R_{1} \approx 0.063 \mathrm{pc}$ and $R_{2} \approx 0.01 \mathrm{pc}$. Substituting the $\mathrm{BH}$ masses and $v_{\mathrm{los}}=3500 \mathrm{~km} \mathrm{~s}^{-1}$ into Equation (4), we get $d=0.27[\sin I \sin (2 \pi t / P)]^{2}$ pc. However, in order to produce the comparable strength of both components and hence two distinct peaks, it requires that the smaller BH (blue component) is $\sim 40$ times more efficient at producing the broad line emission than the larger $\mathrm{BH}$. Furthermore, by comparing the spectrum taken $\sim 1$ yr after (Chornock et al. 2010) with the original SDSS spectrum, it appears that both the blueshifted and redshifted components become slightly weaker, whereas the central component (which would be the classic BLR in the disk emitter scenario) remains almost unchanged. This strengthens the association of a disk emitter origin with the double-peaked feature.

There have been a significant number of double-peaked or highly asymmetric broad line AGNs known in the literature, and large spectroscopic surveys such as SDSS are providing many more (e.g., Strateva et al. 2003; Shen et al. 2010). An example, SDSS J0012-1022, ${ }^{9}$ is shown in Figure 11, whose broad line profile can be well fitted by two components. Note that the velocity splitting of this object is smaller than the FWHM of the redshifted component, and both broad components are reasonably well fitted by a single Gaussian. If it is of a binary origin, its separation must be large enough such that the two BLRs are mostly distinct. Even though its profile is not obviously double-peaked, it still stands out as a promising binary candidate. It would be interesting to perform spectral monitoring and reverberation mapping for a large statistical sample of such objects in order to determine their nature.

\subsection{The Frequency of Spectroscopic Binaries}

There is currently some tension between theoretical expectations and observations on the frequency of SMBH binaries: if major mergers of gas-rich galaxies is the triggering mechanism of quasar activity, then the expected binary fraction is very high; on the other hand, the observed frequency of binary SMBHs is less than a few percent beyond pc scales, and much lower on pc to sub-pc scales. We can parameterize the "observable" binary

\footnotetext{
9 Strictly speaking, this is not a double-peaked profile, but an asymmetric profile with an extended red wing. Nevertheless, it was included in the double-peaked Balmer line AGN sample in Strateva et al. (2003).
}

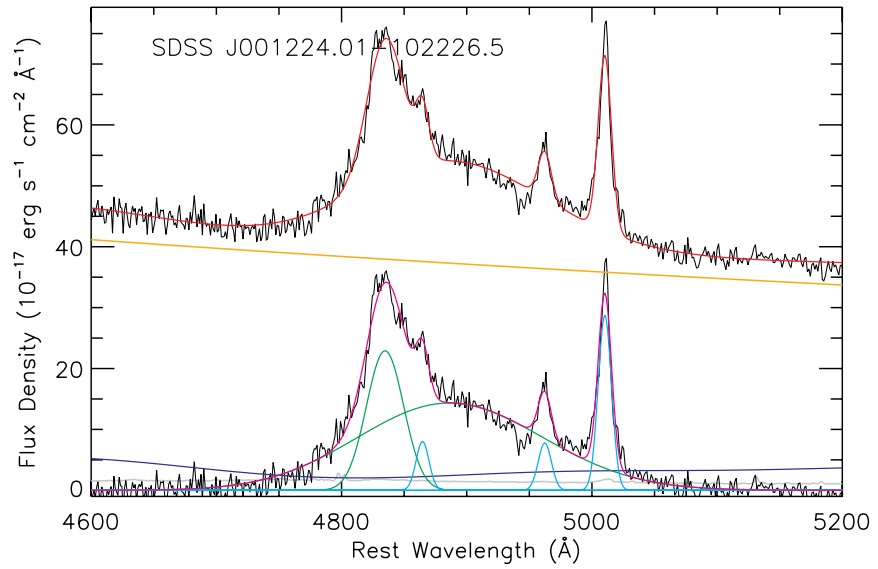

Figure 11. Example of double-peaked broad line AGNs from SDSS. The black lines show the original SDSS spectrum (upper) and the continuum/ iron flux subtracted spectrum (bottom). The red and magenta lines show the overall model fits. The orange and blue lines are the power-law continuum and iron template fits. The three Gaussian components in cyan are the narrow lines $\mathrm{H} \beta$, [O III] $\lambda 4959$, and [O III] $\lambda 5007$ fixed to have the same redshifts and line widths. The two green Gaussian components are for the doublepeaked broad line profile, which are separated by $\sim 3000 \mathrm{~km} \mathrm{~s}^{-1}$, and have FWHMs $\sim 2200 \mathrm{~km} \mathrm{~s}^{-1}$ and $\sim 10,000 \mathrm{~km} \mathrm{~s}^{-1}$ for the blueshifted and redshifted components, respectively. This object and many others will be good candidates for spectral monitoring and reverberation mapping programs.

(A color version of this figure is available in the online journal.)

fraction at different stages as the product of several factors:

$$
f_{\text {obs }}=f_{\text {bin }} \times f_{\text {active }} \times f_{\text {geo }} \times f_{\text {tech }} \times\left(\tau_{\text {phase }} / \tau_{\mathrm{QSO}}\right)
$$

where $f_{\text {bin }}$ is the intrinsic binary fraction $\left(f_{\text {bin }} \approx 1\right.$ in the merger hypothesis for quasar activity), $f_{\text {active }}$ is the probability of both BHs being active, $f_{\text {geo }}$ is the observable fraction due to orientational effects (inclination and orbital phase), $f_{\text {tech }}$ is the observable fraction due to the specific technique used (i.e., spectroscopic methods or spatially resolved imaging) which depends on the data quality of the observing program and folds in the complications associated with the emission-line region geometry, $\tau_{\text {phase }}$ is the time span during the specific stage of binary evolution, and $\tau_{\mathrm{QSO}}$ is the lifetime of quasars. All these quantities except for $\tau_{\mathrm{QSO}}$ are functions of the evolutionary stage of the binary.

In this paper, we have focused on the broad line diagnosis technique, which are for parsec to sub-parsec binaries before the binary enters the gravitational wave dominated regime. Therefore, we expect $f_{\text {active }} \approx 1$ since in gas-rich mergers a nuclear gas disk on pc to $100 \mathrm{pc}$ scales may form and feed both BHs (e.g., Mayer et al. 2007). For the effects of random orientations and orbital phases, it is reasonable to adopt $f_{\text {geo }} \lesssim 0.25$, since nearly edge-on systems with radial motions are most likely to be detected. $f_{\text {tech }}$ is difficult to quantify without a dedicated program and related Monte-Carlo simulations, but it is unlikely that $f_{\text {tech }}$ is close to unity. Bearing in mind the large uncertainties, the time span during this stage can be estimated as $\sim 10^{5}-10^{7}$ yr if gas drag is the dominant mechanism that shrinks the binary orbit (e.g., Escala et al. 2005; Dotti et al. 2006; Mayer et al. 2007). The quasar lifetime is not very well constrained and typical values are $\tau_{\mathrm{QSO}} \sim 10^{7}-10^{8} \mathrm{yr}$. Taken together, the fraction of observable parsec to sub-parsec binaries based on broad line diagnosis is less than a few percent. This is still higher than the frequency of the known parsec to subparsec binary candidates, but lower than the frequency of known double-peaked broad line AGNs (e.g., Strateva et al. 2003; 
Gezari et al. 2007, and references therein). Of course, these are very crude estimates and measuring the actual observed frequency will serve an important role of testing theoretical models of binary formation and evolution (e.g., Volonteri et al. 2009).

We thank the anonymous referee for constructive comments that improved the manuscript. Y.S. acknowledges support from a Clay Postdoctoral Fellowship through the Smithsonian Astrophysical Observatory (SAO). This work was supported in part by NSF grant AST-0907890 and NASA grants NNX08AL43G and NNA09DB30A (A.L.).

\section{REFERENCES}

Bianchi, S., Chiaberge, M., Piconcelli, E., Guainazzi, M., \& Matt, G. 2008, MNRAS, 386, 105

Bogdanović, T., Smith, B. D., Sigurdsson, S., \& Eracleous, M. 2008, ApJS, 174, 455

Bonning, E. W., Shields, G. A., \& Salviander, S. 2007, ApJ, 666, L13

Boroson, T. A., \& Lauer, T. R. 2009, Nature, 458, 53

Chen, K., Halpern, J. P., \& Filippenko, A. V. 1989, ApJ, 339, 742

Chornock, R., et al. 2010, ApJ, 709, L39

Colpi, M., \& Dotti, M. 2009, arXiv:0906.4339

Comerford, J. M., Griffith, R. L., Gerke, B. F., Cooper, M. C., Newman, J. A., Davis, M., \& Stern, D. 2009a, ApJ, 702, L82

Comerford, J. M., et al. 2009b, ApJ, 698, 956

Cuadra, J., Armitage, P. J., Alexander, R. D., \& Begelman, M. C. 2009, MNRAS, 393,1423

Decarli, R., et al. 2009, ApJ, 703, L76

Denney, K. D., et al. 2009, ApJ, 704, L80

Dietrich, M., et al. 1998, ApJS, 115, 185

Dotti, M., Colpi, M., \& Haardt, F. 2006, MNRAS, 367, 103

Dotti, M., Colpi, M., Haardt, F., \& Mayer, L. 2007, MNRAS, 379, 956

Eracleous, M., \& Halpern, J. P. 1994, ApJS, 90, 1

Eracleous, M., Halpern, J. P., Gilbert, A. M., Newman, J. A., \& Filippenko, A. V. 1997, ApJ, 490, 216

Eracleous, M., Livio, M., Halpern, J. P., \& Storchi-Bergmann, T. 1995, ApJ, 438,610

Escala, A., Larson, R. B., Coppi, P. S., \& Mardones, D. 2005, ApJ, 630, 152

Gaskell, C. M. 1983, in Liege International Astrophysical Colloquia, Vol. 24, ed. J.-P. Swings (Cointe-Ougree: Universite de Liege), 473

Gaskell, C. M. 2010, Nature, 463, 1

Gezari, S., Halpern, J. P., \& Eracleous, M. 2007, ApJS, 169, 167

Heckman, T. M., Miley, G. K., van Breugel, W. J. M., \& Butcher, H. R. 1981, ApJ, 247, 403

Horne, K., Peterson, B. M., Collier, S. J, \& Netzer, H. 2004, PASP, 116, 465
Kaspi, S., Brandt, W. N., Maoz, D., Netzer, H., Schneider, D. P., \& Shemmer, O. 2007, ApJ, 659, 997

Kaspi, S., Smith, P. S., Netzer, H., Maoz, D., Jannuzi, B. T., \& Giveon, U. 2000, ApJ, 533, 631

Komossa, S., Burwitz, V., Hasinger, G., Predehl, P., Kaastra, J. S., \& Ikebe, Y. 2003, ApJ, 582, L15

Komossa, S., Zhou, H., \& Lu, H. 2008, ApJ, 678, L81

Lauer, T. R., \& Boroson, T. A. 2009, ApJ, 703, 930

Liu, X., Greene, J. E., Shen, Y., \& Strauss, M. A. 2010a, ApJ, 715, L30

Liu, X., Shen, Y., Strauss, M. A., \& Greene, J. E. 2010b, ApJ, 708, 427

Loeb, A. 2010, Phys. Rev. D, 81, 047503

Luo, B., et al. 2009, ApJ, 695, 1227

Mayer, L., Kazantzidis, S., Madau, P., Colpi, M., Quinn, T., \& Wadsley, J. 2007, Science, 316, 1874

McLure, R. J., \& Jarvis, M. J. 2002, MNRAS, 337, 109

Murray, C. D., \& Dermott, S. F. 1999, in Solar System Dynamics, ed. C. D. Murray \& S. F. Dermott (Cambridge: Cambridge Univ. Press)

Onken, C. A., Ferrarese, L., Merritt, D., Peterson, B. M., Pogge, R. W., Vestergaard, M., \& Wandel, A. 2004, ApJ, 615, 645

Peterson, B. M. (ed.) 1997, An Introduction to Active Galactic Nuclei (Cambridge: Cambridge Univ. Press)

Peterson, B. M. 2010, in IAU Symp. 267, Co-evolution of Central Black Holes and Galaxies, ed. B. Peterson, R. Somerville, \& T. Storchi-Bergmann (Cambridge: Cambridge Univ. Press), 151

Peterson, B. M., Korista, K. T., \& Cota, S. A. 1987, ApJ, 312, L1

Peterson, B. M., \& Wandel, A. 2000, ApJ, 540, L13

Peterson, B. M., et al. 2004, ApJ, 613, 682

Popovic, L. C., Mediavilla, E. G., \& Pavlovic, R. 2000, Serb. Astron. J., 162, 1

Press, W. H., Teukolsky, S. A., Vetterling, W. T., \& Flannery, B. P. 1992, Numerical Recipes in FORTRAN. The Art of Scientific Computing (Cambridge: Cambridge Univ. Press)

Rodriguez, C., Taylor, G. B., Zavala, R. T., Peck, A. B., Pollack, L. K., \& Romani, R. W. 2006, ApJ, 646, 49

Sergeev, S. G., Pronik, V. I., Sergeeva, E. A., \& Malkov, Y. F. 1999, ApJS, 121, 159

Shen, Y., Greene, J. E., Strauss, M. A., Richards, G. T., \& Schneider, D. P. 2008, ApJ, 680, 169

Shen, Y., et al. 2010, arXiv:1006.5178

Shields, G. A., et al. 2009, ApJ, 707, 936

Smith, K. L., Shields, G. A., Bonning, E. W., McMullen, C. C., \& Salviander, S. 2009, ApJ, 716, 866

Strateva, I. V., et al. 2003, AJ, 126, 1720

Tang, S., \& Grindlay, J. 2009, ApJ, 704, 1189

Volonteri, M., Miller, J. M., \& Dotti, M. 2009, ApJ, 703L, 86

Wang, J., Chen, Y., Hu, C., Mao, W., Zhang, S., \& Bian, W. 2009, ApJ, 705 , L76

Wrobel, J. M., \& Laor, A. 2009, ApJ, 699, L22

Xu, D., \& Komossa, S. 2009, ApJ, 705, L20

Zhou, H., Wang, T., Zhang, X., Dong, X., \& Li, C. 2004, ApJ, 604, L33 